\title{
Epidemiological trends, predictive factors, and projection of tooth loss in Germany 1997-2030: part I. missing teeth in adults and seniors
}

\author{
A. Rainer Jordan ${ }^{1} \cdot$ H. Stark ${ }^{2} \cdot$ I. Nitschke ${ }^{3} \cdot$ W. Micheelis ${ }^{1} \cdot$ F. Schwendicke ${ }^{4}$ \\ Received: 27 March 2019 / Accepted: 19 March 2020 / Published online: 21 November 2020 \\ (C) The Author(s) 2020
}

\begin{abstract}
Objective This is the first part of a report on tooth loss in Germany 1997-2030. Here, we describe trends in the prevalence of tooth loss in adults and seniors 1997-2014, assess predictive factors for tooth loss and projected it into 2030.

Material and methods Data of the cross-sectional, multi-center, nationally representative German Oral Health Studies of 1997, 2005, and 2014 were used. Age, sex, educational level, smoking status, and the cohort were used for ordinary least square regression to assess the association of predictors with tooth loss (missing teeth, MT). The yielded regression coefficients were used to predict tooth loss in 2030.

Results Compared with 1997, the mean MT in adults (35-44 years old) in 2030 was predicted to decrease by two-thirds to 1.3. The prevalence of tooth loss (MT > 0) will decrease by $72 \%$ from 1997 to 2030. In 2030, half of the population of adults will not exhibit any tooth loss. Compared with 1997, the mean MT among seniors (65-74 years old) will decline to 5.6 teeth (i. e. twothirds reduction) until 2030. Prevalence of tooth loss will be halved by 2030, and approximately one-third of this age group will not exhibit any tooth loss.

Conclusions Based on the model used, the trend of a robust decline in tooth loss will become more dynamic by the year 2030. As a result, every second adult will have experienced no tooth loss at all in 2030, and seniors will possess more teeth than they have previously lost.

Clinical relevance This study presents the trends of tooth loss in Germany for a period of three decades. It provides clinically relevant data for health care planning by 2030 .
\end{abstract}

Keywords Dental caries $\cdot$ Dentistry $\cdot$ Epidemiology $\cdot$ Periodontitis $\cdot$ Tooth loss

A. Rainer Jordan

r.jordan@idz.institute

1 Institute of German Dentists (IDZ), Universitaetsstraße 73, D-50931 Cologne, Germany

2 Department of Prosthodontics, Preclinical Education, and Dental Materials Science, Rhenish Friedrich Wilhelms University of Bonn, Bonn, Germany

3 Clinic for Geriatric and Special Care Dentistry, Center of Dental Medicine, University of Zurich, Zurich, Switzerland

4 Department of Operative and Preventive Dentistry, Charité Universitätsmedizin Berlin, Berlin, Germany

\section{Introduction}

Dental caries and periodontal diseases are the most common oral diseases and the main cause of tooth loss [1]. From an epidemiological point of view, caries experience has been continuously declining since the 1990s in Germany, both in children, adolescents, and among adults, which has a subsequent impact on the rate of tooth loss, especially in the first half of life [2]. The number of periodontally affected teeth, however, has been increasing in parallel [3], with the majority of these teeth being retained long term, also into higher age. Concomitantly, the older population segment is growing, while younger groups are shrinking. Overall, there is a strong indication for oral health gains in a large segment of the life curve on an individual level, but a compression of morbidity in 
elderly individuals and also older population segments [4].

Tooth loss is a very robust oral-epidemiological health marker, as it is relatively easy to measure. It is highly relevant to patients. Tooth loss is thus an epidemiological key variable and is also used to assess the quality of a dental healthcare [5]. Tooth loss exceeding a certain number of teeth has a significant impact on chewing function, nutrition, speech, and esthetics. This is even more true for complete tooth loss (edentulism), which is the final event of tooth loss and has a specific impact on both quality of life and therapy options. Edentulism is, at least in many high-income countries, a phenomenon of the older population [6-8].

There are only a few longitudinal studies that can identify trends in tooth loss and associate them with trends in caries experience and prevalence, or extent and severity of periodontal disease [9-12]. Cross-sectional surveys may be used as an alternative for identifying trends, but require the consideration of possible cohort effects. Such crosssectional studies are frequently used for epidemiological population monitoring and for deducing interventions in healthcare planning.

The present study is the first part of a report on tooth loss in Germany. The aim of this study was [1] to illustrate the past trends in tooth loss and edentulism in Germany in the past quarter century on the basis of data from three waves of German oral health studies, [2] to evaluate factors that can predict tooth loss and edentulism at the population level, and [3] to use these predictors to forecast tooth loss and edentulism in the year 2030. This first report is devoted to tooth loss in particular. This is clinically relevant for all adult age groups. In a second paper, we report on edentulism in more detail, focusing on seniors (Fig. 1).

\section{Materials and methods}

The Transparent Reporting of a multivariable prediction model for Individual Prognosis Or Diagnosis (TRIPOD) initiative developed a transparent set of suggestions for the reporting of studies developing, validating, or updating a prediction model, whether for diagnostic or prognostic purposes. The reporting of this study (part 1 and part 2) follows the TRIPOD statement [10].

\section{Source of data}

This study involved pseudonymous data from three waves of the German Oral Health Studies (Deutsche Mundgesundheitsstudien, DMS): DMS III from 1997 [13], DMS IV from 2005 [14], and DMS V from 2014 [15, 16]. The studies were conducted by the Institute of German Dentists (Institut der Deutschen Zahnärzte, IDZ). DMS are cross-sectional, multi-center, nationally representative, clinical and socio-epidemiological studies to investigate the oral health status and behavior of the German resident population in several age cohorts.

\section{Participants}

Study participants were drawn from local residents' registration offices out of 90 randomly selected communities. A disproportional sample point selection was used, resulting in 60 study sample points in the Western federal states of Germany and 30 study sample points in the Eastern states with post hoc re-dressing. Stratification was performed according to federal states and levels of urbanization. Our data analysis accounted for this complex sample and the associated weighting (see below). The names and
Fig. 1 Mean missing teeth (MT) in different age cohorts and waves of the German Oral Health Studies (DMS)

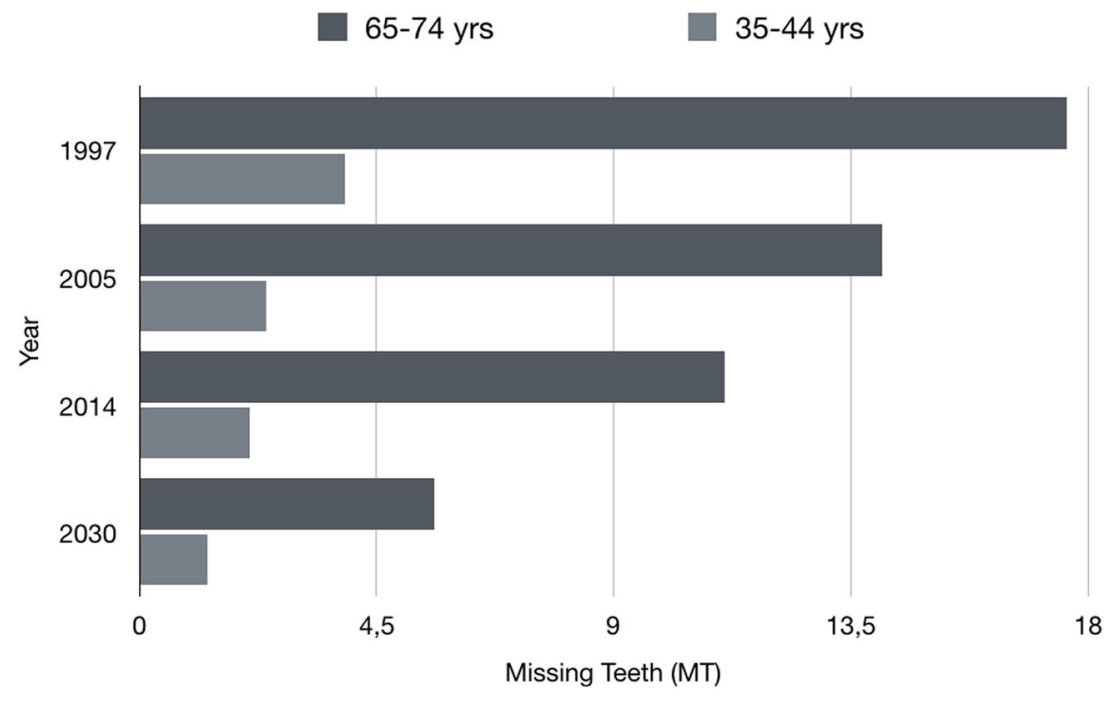


addresses of the study participants to be invited were drawn from the registration files of the local residents' registration offices. Participants were drawn from three age groups: 12 years old, 35 to 44 years old, and 65 to 74 years old; and, only in 2014,75 to 100 years old.

\section{Sample size}

Per DMS wave, it was the aim to include 1000 subjects (net) per each age group into the study. To achieve this, 2000 participants were sampled per age group. For the DMS III, 3065 participants were included (response rate of 63.6\%); for DMS IV and $\mathrm{V}$, these numbers were $4631(63.1 \%)$ and 4609 $(50.1 \%)$, respectively. Empirical non-responder analyses were conducted to compare the socio-dental characteristics of responders with the target population according to gender, educational level, dental visiting patterns, and dental/ prosthodontic status. Non-response bias was found to be minimal.

Here, only the age groups of 35 to 44 years old (younger adults) and of 65 to 74 years old (younger seniors) are reported. This was done, as the older senior group was not available in 1997 and 2005, and as tooth loss was virtually absent in the 12-year-old age group (2.1\% in DMS III, 1997; $1.0 \%$ in DMS IV, 2005; and 1.2\% in DMS V, 2014). Overall, 2546 younger adults (DMS III 1997, 655; DMS IV 2005, 925; DMS V 2014, 966) and 3449 younger seniors (DMS III 1997, 1367; DMS IV 2005, 1040; DMS V 2014, 1042) were included in this study.

\section{Dental examinations and outcomes}

The socio-scientific survey and the oral examination were carried out at venues of the local health authorities. A paper questionnaire was completed by the subjects before clinical examination. The following clinical parameters were assessed: tooth loss, dental caries, periodontal disease, prosthodontic status, developmental and acquired dental hard tissue, and mucosal lesions. To ensure reproducibility, the dental investigators were trained and calibrated by experts. Multiple reliability checks were performed throughout the field phases.

For the present study, the outcome was tooth loss, expressed as extent (number of missing teeth, MT primary outcome) and prevalence (\%MT $>0$ secondary outcome). Third molars were excluded for this analysis. Inter-rater reliability (Kendall's tau) for MT between study dentist examiners as compared to a master dentist was 0.99 (DMS III, 1997), 0.89 (DMS IV, 2005), and 0.95 (DMS $\mathrm{V}, 2014)$. Data on edentulism can be found in the second part of this paper.

\section{Predictor variables}

Predictor variables were recorded at the beginning of the participants' examination using self-administered validated questionnaires. Note that for the present study, only those predictors that were concurrently available in socio-demographic projections for 2030 were used. The following predictor variables were used: (1) age in years; (2) sex; (3) educational level as low, middle, or high; (4) smoking status as never, former, and current; and (5) the cohort (coded via study wave as dummy variable DMS III, 1997; DMS IV, 2005; DMS V, 2014).

\section{Missing data}

Missing predictor variables occurred very rarely $(<2 \%$ of cases, $<1 \%$ of entries) and were handled as missing at random, with exclusion of the respective subject from the model. Multiple imputation using the simple random imputation method was performed in a sensitivity analysis but yielded very similar coefficients allowing this procedure.

\section{Statistical analysis}

Age cohort regression models were created to examine the association between predictor variables and the primary outcome (MT), and to deduce future trends [17]. Ordinary least square linear regression models were used for this purpose. All predictors were entered jointly (only multivariable models were used). A number of interaction terms were tested, without the model being more appropriate. Regression coefficients, expressing the mean difference in MT, and 95\% confidence intervals (CI), were used to present the risk of tooth loss. Differences were considered to be statistically significant at $p<0.05$. Statistical analysis was performed using SPSS 22 (IBM, Armonk, USA) plugged into the open-source software R (version 3.1, the R foundation).

\section{Model validation}

Model validation was performed via split-sample validation comprising two random half of each cohort (stratified by age group). The coefficients yielded from the model developed in one half were used to predict the number of lost teeth in the other half.

\section{Projection 2030}

To make projections of MT in 2030, the yielded regression coefficients from the validated models were used and applied them to the predicted population of younger adults and younger seniors in 2030. These population age ranges were chosen 
as tooth loss was expected to occur in relevant numbers from adults onwards. To do so, predictor data were collected from a number of sources:

1 Predicted demographic data were drawn from the national statistical office [18].

2 Sex proportions in these age groups were estimated for 35 to 44 years old at $48 \%$ female and $52 \%$ male in 2030 , and for 65 to 74 years old exactly the other way round; $52 \%$ female and $48 \%$ male [18].

3 Socio-educational status was assumed to be carried forward from the population 35-44 years in 1997, as educational status to change greatly after that age was not assumed, with $30 \%, 41 \%$, and $29 \%$ of individuals being in the low, medium, and high social group, respectively. For the future younger adults, the socio-educational status to the same as in 2014 was assumed.

4 Smoking status was derived from predictions made by the WHO, with stratification for sex [19]. Thirty-two percent of current smokers were assumed, $19 \%$ former smokers in the younger adult group, and 29\% current and 19\% former smokers in the younger senior group.

Spreadsheet-based Monte Carlo simulations were used to predict MT for a simulated population of 3000 individuals (as could be expected to be drawn by the 2030 wave of the DMS 7). Parameter uncertainty was introduced by randomly sampling variables from a binomial distribution. Each population was modelled 100 times, yielding mean MT and 95\% CI. YASAIw (University of Washington) plugged into Microsoft Excel (Microsoft, Redwood, USA) was used for this modeling.

Population level estimates were calculated using past and predicted demographic data from the national bureau of statistics [18] with absolute and relative differences being calculated over the 33-year period (1997-2030). The population level estimates were further divided into different sex and socio-educational strata as well as according to smoking status.

\section{Results}

The overall characteristics of the sampled cohorts in 1997, 2005, and 2014 are displayed in Table 1. While sex proportions remained relatively stable in both age groups over time, education level increased. Smoking decreased mainly in younger adults, but not in younger seniors.

MT and the prevalence of tooth loss decreased with time in both age groups. While MT decreased by nearly $50 \%$ in both age groups between 1997 and 2014, the prevalence of tooth loss decreased by $20 \%$ in younger adults, but only by $2 \%$ in younger seniors.

All predictors were significantly associated with MT (Table 2); that are the age per life year, sex, educational level, smoking behavior, and the cohort (DMS wave). With each year of age, MT increased in younger adults by 0.195 teeth and in younger seniors by 0.509 teeth. MT was also higher in females than males, those from low versus medium and high educational level, and in current versus former and never smokers. The model was robust when split-sample coefficients were tested in the other half of the sample (the predicted prevalence in the different waves deviated by $<2 \%$ when compared to reported prevalence).

The yielded coefficients were then applied to project MT and tooth loss prevalence in 2030 (Table 3 ). The projected MT in younger adults was 1.3 and was 5.6 in younger seniors. Predicted prevalence of tooth loss for the year 2030 in younger adults and younger seniors is $50 \%$ and $65 \%$, respectively. In absolute terms, these were 36 and 65 million fewer MT in 2030 compared with 1997, respectively. The reduction in MT was higher in those from a lower than medium and high educational level.

Looking at tooth loss in relation to the dental arch, it is noticeable that the general decrease in tooth loss over time has occurred equally in both age groups and in anterior and posterior teeth, respectively. Interestingly, the anterior tooth loss in younger seniors is more pronounced than in the posterior area, whereas the distribution in younger adults is almost symmetrical (Appendix Table 4).

\section{Discussion}

This study describes trends in tooth loss in adults and younger seniors in Germany as measured by repeated waves of a population-representative cross-sectional survey. In our sample and based on nearly 6000 participants, the strongest predictors for MT (and edentulism, see part II) were the cohort, patients' age, and education level as well as smoking status. In this respect, our findings are in line with previous evaluations of tooth loss $[20,21]$.

In addition to the known risk factors that can lead to tooth loss, so-called dental generations can also be identified which, as a result of cohort effects, orally represent the current state of health technology in dental care. Our findings regarding the different "dental generations" [22] demonstrate the association between the caries decline and tooth loss. The adults sampled into DMS IV and 
Table 1 Characteristics of included subjects in different age cohorts and waves of the German Oral Health Studies (DMS). a 35 to 44 years old, b 65 to 74 years old

\begin{tabular}{|c|c|c|c|c|c|c|c|}
\hline \multirow[t]{2}{*}{ Parameter } & & \multicolumn{2}{|c|}{ DMS III (1997) } & \multicolumn{2}{|c|}{ DMS IV (2005) } & \multicolumn{2}{|c|}{ DMS V (2014) } \\
\hline & & $n$ & $\%$ & $n$ & $\%$ & $n$ & $\%$ \\
\hline \multicolumn{8}{|c|}{ (a) 35 to 44 years old } \\
\hline $\begin{array}{c}\text { Total age } \\
\text { cohort }\end{array}$ & & 655 & 100 & 925 & 100 & 966 & 100 \\
\hline Age (years) & $\begin{array}{l}\text { Mean }(\mathrm{SD}), \\
\text { range }\end{array}$ & $39(3)$ & $\begin{array}{c}35-44 \\
y\end{array}$ & $39(3)$ & $\begin{array}{c}35-44 \\
y\end{array}$ & $40(3)$ & $\begin{array}{c}35-44 \\
y\end{array}$ \\
\hline \multirow[t]{2}{*}{ Sex } & Male & 332 & 50.7 & 471 & 50.9 & 485 & 50.2 \\
\hline & Female & 323 & 49.3 & 454 & 49.1 & 481 & 49.8 \\
\hline \multirow{3}{*}{$\begin{array}{l}\text { Educational } \\
\text { level }\end{array}$} & Low & 195 & 30.0 & 222 & 24.3 & 265 & 26.5 \\
\hline & Medium & 261 & 40.3 & 374 & 40.9 & 350 & 36.3 \\
\hline & High & 192 & 29.6 & 318 & 34.8 & 358 & 37.2 \\
\hline \multirow{3}{*}{$\begin{array}{c}\text { Smoking } \\
\text { pattern }\end{array}$} & Never & 264 & 40.6 & 402 & 43.9 & 425 & 44.1 \\
\hline & Former & 139 & 21.4 & 191 & 20.9 & 249 & 25.9 \\
\hline & Current & 247 & 38.0 & 332 & 35.2 & 289 & 30.0 \\
\hline \multirow[t]{2}{*}{ Tooth loss } & Prevalence $(\%)$ & 502 & 76.6 & 544 & 58.8 & 549 & 56.8 \\
\hline & $\begin{array}{l}\text { Mean MT (SD), } \\
\text { range }\end{array}$ & $\begin{array}{c}3.9(2.8), \\
0-28\end{array}$ & $0-100$ & $\begin{array}{c}2.4(2.8) \\
0-28\end{array}$ & $0-100$ & $\begin{array}{c}2.1(2.9), \\
0-28\end{array}$ & $0-100$ \\
\hline
\end{tabular}

(b) 65 to 74 years old

$\begin{array}{cll}\begin{array}{c}\text { Total age } \\ \text { cohort } \\ \text { Age (years) }\end{array} & \begin{array}{c}\text { Mean (SD), } \\ \text { range }\end{array} & 69(2.7) \\ \text { Sex } & \text { Male } & 578 \\ & \text { Female } & 789 \\ \text { Educational } & \text { Low } & 1031 \\ \text { level } & \text { Medium } & 183 \\ & \text { High } & 145 \\ \text { Smoking } & \text { Never } & 815 \\ \text { pattern } & \text { Former } & 358 \\ & \text { Current } & 183 \\ \text { Tooth loss } & \text { Prevalence (\%) } & 1342 \\ & \text { Mean MT (SD), } & 17.6(9.1), \\ & \text { range } & 0-28\end{array}$

\begin{tabular}{clcll}
\multicolumn{1}{c}{1040} & \multicolumn{3}{c}{1042} \\
$65-74$ & $69(2.7)$ & $65-74$ & $70(2.9)$ & $65-74$ \\
$\mathrm{y}$ & & $\mathrm{y}$ & & $\mathrm{y}$ \\
42.2 & 480 & 46.2 & 489 & 46.9 \\
57.8 & 560 & 53.8 & 553 & 53.1 \\
75.9 & 666 & 65.8 & 642 & 63.9 \\
14.0 & 183 & 18.1 & 190 & 18.9 \\
10.7 & 163 & 16.1 & 173 & 17.2 \\
60.1 & 627 & 62.0 & 545 & 53 \\
26.4 & 305 & 29.9 & 362 & 34.9 \\
13.5 & 88 & 8.6 & 131 & 12.6 \\
98.2 & 1013 & 97.4 & 1004 & 96.3 \\
$0-100$ & $14.1(9.8)$, & $0-100$ & $11.1(9.1)$, & $0-100$ \\
& $0-28$ & & $0-28$ &
\end{tabular}

DMS V $(2005,2014)$ have benefitted from the wide availability of fluoride toothpaste and preventive programs (fluoride varnish and sealant application in dental practice and group prophylaxis in kindergartens and schools) installed in the late 1970 s and 1980s, respectively. In contrast, the adults sampled into DMS III (1997) and the seniors of DMS V had experienced higher caries increments and had been mainly treated restoratively, eventually leading to the final endpoint of the disease, tooth loss. The seniors from DMS III and IV had even entered adulthood with some missing teeth; the extent then increased even further up until age 65-74 years. The three cohorts of the DMS thus represent the "denture," the "filling," and the "fluoride" generation: given the chronic-cumulative nature of dental diseases, the endpoint tooth loss can be used to characterize them.

Edentulism can be considered a special case of tooth loss, namely the state after the final "target event." Despite the closeness of tooth loss and edentulism, there exist clear epidemiological distinctions between these two states. For example, their prevalence is highly different; while tooth loss is highly prevalent in both age groups, edentulism is practically nonexistent among adults. Moreover, the changes observed over time (1997-2014) between both states are remarkable: While the prevalence of tooth loss decreased by one quarter in younger adults (from 77 to $57 \%$ ), it was almost unchanged in younger seniors (from 98 to $96 \%$ ). This was also reflected in trends 
Table 2 Multivariate analysis. a 35 to 44 years old, b 65 to 74 years old (log-likelihood a 438.826, b 790.833; $\mathrm{B}=$ regression coefficient)

\begin{tabular}{lllll}
\hline Parameter & Category & B & $95 \%$ CI & $P$ value \\
\hline (a) 35 to 44 years old & & & & \\
Cohort (ref: 1997) & 2005 & -1.547 & $-5.321 ;-1.305$ & 0.001 \\
& 2014 & -1.758 & $-2.113 ;-1.404$ & $<0.001$ \\
Age & Per year & 0.195 & $0.147 ; 0.243$ & $<0.001$ \\
Gender (ref: male) & Female & 0.365 & $0.084 ; 0.645$ & 0.011 \\
Educational level (ref: low) & Medium & -1.236 & $-1.596 ;-0.875$ & $<0.001$ \\
& High & -2.250 & $-2.625 ;-1.875$ & $<0.001$ \\
Smoking (ref: never) & Former & 0.186 & $-0.174 ; 0.546$ & 0.311 \\
& Current & 1.473 & $1.146 ; 1.800$ & $<0.001$ \\
(b) 65 to 74 years old & & & & $<0.001$ \\
Cohort (ref: 1997) & 2005 & -3.066 & $-3.793 ;-2.340$ & $<0.001$ \\
& 2014 & -6.028 & $-6.718 ;-5.337$ & $<0.001$ \\
Age & Per year & 0.509 & $0.406 ; 0.612$ & $<0.001$ \\
Gender (ref: male) & Female & 1.806 & $1.175 ; 2.437$ & $<0.001$ \\
Educational level (ref: low) & Medium & -4.020 & $-4.790 ;-3.250$ & $<0.001$ \\
& High & -5.717 & $-6.526 ;-4.909$ & $<0.000$ \\
Smoking (ref: never) & Former & 2.111 & $1.414 ; 2.808$ & $<0.001$ \\
& Current & 5.403 & $4.568 ; 6.349$ &
\end{tabular}

on edentulism, which halved (from 22 to $12 \%$ ) in younger seniors but remained stable (from 1 to $0.8 \%$ ) in younger adults (see part II of this paper). It is thus relevant to evaluate both states separately, also given the different treatment needs and subjective impact emanating from them.

Our findings can be contrasted with that from other studies. In accordance with our data, one Finnish study reported a significant decrease of MT between 1978 and 1997 , together with a decrease in prevalence from 80 to $60 \%$. The number of adults with reduced dentition (MT > 6) decreased from 26 to $14 \%$ with time [23, 24]. A Swedish study reported that between 1975 and 1997, the number of adults with tooth loss decreased from 23 to $10 \%$, whereas this number remained almost constantly among younger seniors (at 25\%) [25]. Globally, severe tooth loss in the permanent dentition has been nearly halved since 1990 from almost 40 to $20 \%$, again in line with our data [26].

This study has a number of strengths and limitations. One strength is that the available data are highly intrinsically consistent, as all three examinations were developed, collected, and evaluated by the same study center. Second, the used analytic method for the projection had been tested, validated, and applied to project edentulism, too (see part II); the present study supplements this method from a binary to a continuous outcome and helps to describe the oral health status in Germany in more depth. As limitation, it was not possible to draw testable, causal connections from our analysis given the (repeated) crosssectional character of the underlying data. Thus, no analysis as to the reasons of tooth loss and the associated pathways, involving dental caries and periodontitis, was possible. Rather, this is a descriptive analysis and projection. The future wave of the DMS in 2021 will, for the first time, allow re-examining the participants of the DMS $\mathrm{V}$ and will thus be the first population-representative longitudinal oral health observation in Germany. Second, only a limited number of predictor variables were used in our study, mainly dictated by the availability of predictions of these variables in 2030, whereby the percentage of explained variance (R-squared) fluctuates, depending on the incorporated DMS waves, between $7.8 \%$ and $13.5 \%$ for the adult group, and between $7.7 \%$ and $8.9 \%$ for the senior group. These $\mathrm{R}^{2}$ values thus appear moderately strong at best. While it was thus not able to reliably predict the individual tooth loss, the findings (from the validation) clearly demonstrate the ability to predict tooth loss on population level. In any case, our projection is only as robust as the predictions of these variables are, and prone to unforeseen social or demographic but also economic etc. changes. Third, our model was validated only in the population it was developed in using the split-sample technique; it will most likely be less accurate when applied to other populations. Thus, a different morbidity dynamic with regard to tooth loss in Germany could emerge in the medium term if global migration 


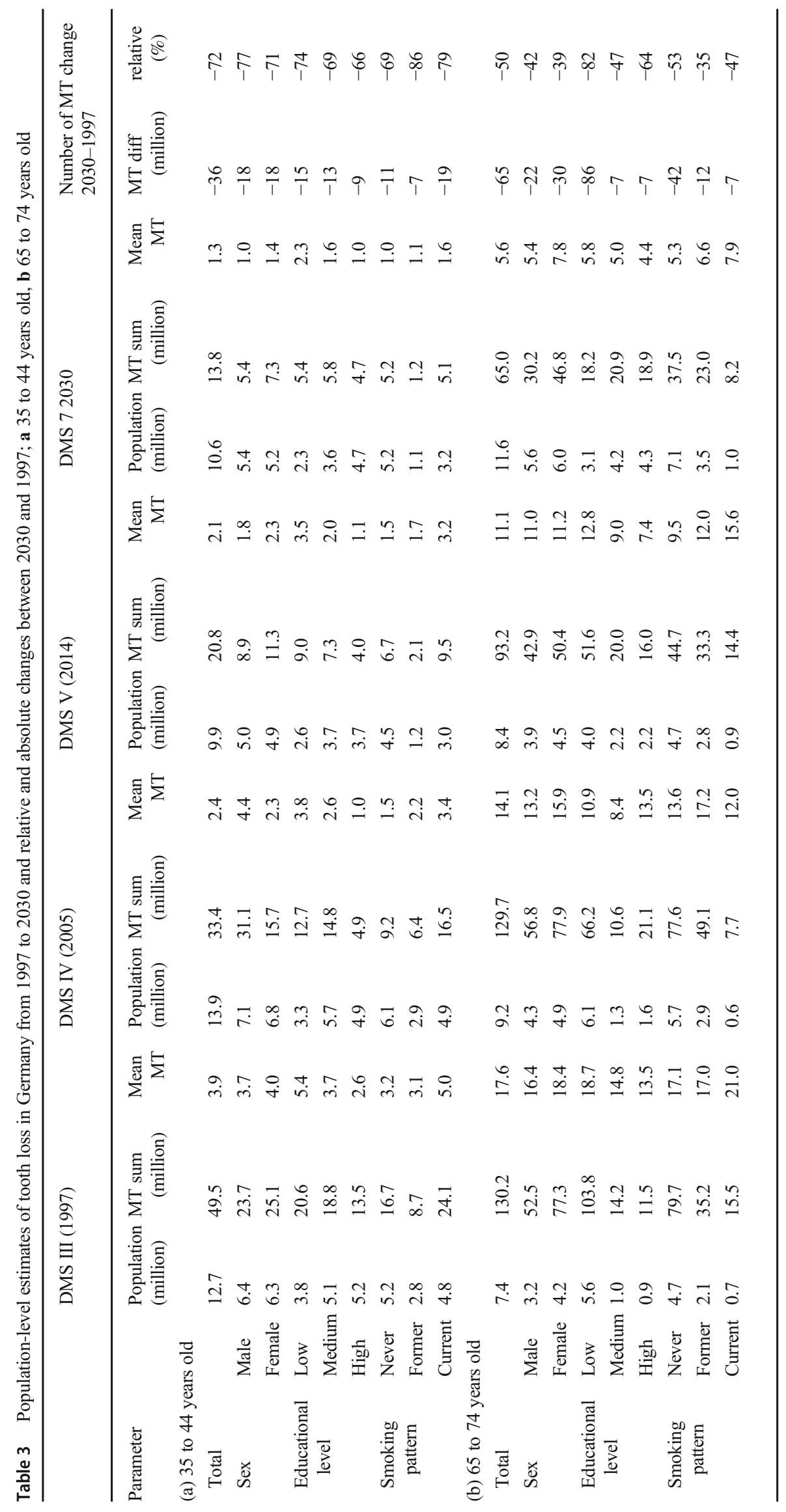


continues and, in addition, Germany will compensate for the lack of skilled workers in the future through a targeted immigration policy. An epidemiological study of oral health and access of older migrants in Germany has shown that the number of missing teeth of migrants is only insignificantly higher than that of non-migrants of the same age (70.2 yrs. of age) (MT migrants 14.4 vs. MT non-migrants $12.6 ; p=0.301)(27)$. However, as this is a first orienting study, it is not possible to assess with certainty how tooth loss will develop in Germany in the future in times of global migration and immigration. Fourth, our primary outcome was the extent of tooth loss (MT), not the prevalence. The model for the prediction of prevalence was not validated; the projected prevalence should thus be regarded with caution. Last, only two selected age groups were analyzed. Our absolute estimates are not able to catch the burden of tooth loss in the whole German population. Given the expected demographic shift and the only recently measured oral health status of older seniors (in the DMS V), assessing and projecting tooth loss in the very old seems highly relevant, too, and should be performed in future studies. Especially in this age group, linking tooth loss data with general health or further socio-demographic parameters is of interest, also to gauge causal (bi-directional) pathways and to determine the subjective impact of (also non-severe) tooth loss (and tooth replacement). The Study of Health in Pomerania (SHiP), for example, links tooth loss and further general health data [27]. Eventually, a further triangulation with qualitative sociological research might also assist a deeper interpretation of tooth loss findings.

\section{Conclusion}

In conclusion, and within the limitations of this study, a robust and continuous decrease in tooth loss in the German population has occurred between 1997 and 2014. Based on our projection, this trend will become more dynamic by the year 2030 , with around $50 \%$ of adults not having experienced any tooth loss in 2030, while young seniors will possess more teeth than they have previously lost. This decrease is likely to reduce the need for tooth replacement treatments, while the challenges of retaining more teeth for longer (for example, with regard to periodontal diseases and root caries) will need to be actively addressed.

Acknowledgments The study was coordinated by Kantar Health $\mathrm{GmbH}$, Munich (Germany) after a call for tenders published in the information system for European public procurement on May 31st, 2013 (2013-073518). We hereby acknowledge the contributions of the field and research staff of the "DMS V Surveillance Investigators' Group".
DMS V surveillance investigators group Principal investigators and Steering Committee: A. Rainer Jordan, Wolfgang Micheelis

Steering Committee assistant: Inge Bayer

Field project management: Constanze Bodechtel, Ursula Reis

Project management assistant: Linda Scharf

Operations: Kantar Health GmbH, Munich (Germany) and TNS

Infratest, Munich (Germany)

Coordinating Center: Institute of German Dentists (IDZ), Cologne (Germany)

Development of questionnaires and manual of operations: A. Rainer Jordan, Katrin Hertrampf, Thomas Hoffmann, Thomas Kocher, Wolfgang Micheelis, Ina Nitschke, Ulrich Schiffner, Helmut Stark

Data management and statistical team: Sarah Noffz, Birte Holtfreter

Data privacy protection: Thomas Bachmann

Online data entry software (DentaSoft V): Jörg Eschenlohr

Field supervisor: Thomas Rappold, Elisabeth Füßl-Grünig

Field monitor: Elisabeth Füß1-Grünig, Constanze Bodechtel

Field contact persons: Dieter Baumert, Peter Brietsche, Markus Nispel, Joachim Schulze

Field interviewers: Gülay Aykal, Beate Bohnke-Schulz, Regine Dirbach, Martin Engler, Jutta Gesk, Bernd Habersaat, Rita Schleypen

Dental investigators: Kerstin Groß, Nele Kettler, Johannes Löw, Nikola Johanna Sprehe, Detlef Joachim Weimar

Authors' contributions All authors listed in the paper have contributed sufficiently to fulfill the criteria for authorship according to ICMJE guidelines. All authors read and approved the final manuscript. RAJ is the principal investigator and writing the manuscript. HS is a member of the DMS V expert advisory board and responsible for the development of the prosthetic dentistry clinical examinations and co-writing the manuscript. IN is a member of the DMS $\mathrm{V}$ expert advisory board and responsible for the development of the gerodontal clinical examinations and co-writing the manuscript. WM is a DMS V sociologic counselor and co-writing the manuscript. FS is a co-principal investigator for the German 2030 dental supply atlas and responsible for statistical analysis and writing the manuscript.

Funding information The German Oral Health Studies are proudly financed by the German dental profession via the German Dental Association (Bundeszahnärztekammer - Arbeitsgemeinschaft der Deutschen Zahnärztekammern - (BZÄK) e. V.) and the National Association of Statutory Health Insurance Dentists (Kassenzahnärztliche Bundesvereinigung (KZBV) KdöR). However, the writing group is solely responsible for the contents of this paper.

\section{Compliance with ethical standards}

Conflict of interest The authors declare that they have no conflict of interest.

Ethical approval All procedures performed in DMS studies involving human participants were in accordance with the ethical standards of the institutional research committee and with the 1964 Helsinki declaration and its later amendments or comparable ethical standards.

Informed consent Informed consent was obtained from all individual participants included in the study. 


\section{Appendix}

Table 4 Population-level distribution of tooth loss (MT) in Germany from 1997 to 2014; (a) 35- to 44-year-olds, (b) 65- to 74-year-olds.

\begin{tabular}{|c|c|c|c|c|c|c|c|c|c|c|}
\hline & & \multirow[t]{2}{*}{ Mean MT } & \multicolumn{8}{|c|}{ Prevalence (\%) } \\
\hline & & & $\mathrm{MT}=0$ & $\mathrm{MT}=1$ & $\mathrm{MT}=2$ & $\mathrm{MT}=3$ & $\mathrm{MT}=4$ & $\mathrm{MT}=5$ & $\mathrm{MT}=6$ & $\mathrm{MT}=7$ \\
\hline \multicolumn{11}{|c|}{ (a) 35 to 44 years old } \\
\hline \multirow[t]{3}{*}{ Anterior teeth } & DMS III (1997) & 2.2 & 40.5 & 18.2 & 12.5 & 6.6 & 8.7 & 3.8 & 1.2 & 1.4 \\
\hline & DMS IV (2005) & 1.4 & 52.8 & 17.5 & 12.0 & 6.4 & 5.0 & 1.8 & 1.0 & 0.6 \\
\hline & DMS V (2014) & 1.0 & 62.8 & 14.2 & 10.2 & 3.9 & 5.9 & .9 & .6 & 0 \\
\hline \multirow[t]{3}{*}{ Posterior teeth } & DMS III (1997) & 2.13 & 29.0 & 19.5 & 16.9 & 13.0 & 9.8 & 4.3 & 3.1 & 2.4 \\
\hline & DMS IV (2005 & 1.46 & 43.4 & 18.9 & 14.5 & 10.2 & 5.7 & 3.0 & 1.5 & 1.2 \\
\hline & DMS V (2014) & .93 & 59.1 & 16.1 & 11.6 & 6.0 & 4.0 & 1.0 & .7 & .4 \\
\hline \multicolumn{11}{|c|}{ (b) 65 to 74 years old } \\
\hline \multirow[t]{3}{*}{ Anterior teeth } & DMS III (1997) & 11.7 & 4.7 & 5.3 & 4.6 & 5.1 & 5.0 & 3.0 & 3.7 & 3.7 \\
\hline & DMS IV (2005) & 9.0 & 9.9 & 10.1 & 8.5 & 6.4 & 5.7 & 4.9 & 3.5 & 3.6 \\
\hline & DMS V (2014) & 6.3 & 16.7 & 12.6 & 12.8 & 8.7 & 6.7 & 4.8 & 3.9 & 3.6 \\
\hline \multirow[t]{3}{*}{ Posterior teeth } & DMS III (1997) & 6.4 & 2.3 & 2.9 & 2.9 & 4.5 & 7.4 & 8.6 & 9.9 & 10.9 \\
\hline & DMS IV (2005) & 5.4 & 5.1 & 5.5 & 8.0 & 8.8 & 8.1 & 9.8 & 1.0 & 8.1 \\
\hline & DMS V (2014) & 4.4 & 8.4 & 9.8 & 8.9 & 11.2 & 12.7 & 11.5 & 8.6 & 8.0 \\
\hline
\end{tabular}

Open Access This article is licensed under a Creative Commons Attribution 4.0 International License, which permits use, sharing, adaptation, distribution and reproduction in any medium or format, as long as you give appropriate credit to the original author(s) and the source, provide a link to the Creative Commons licence, and indicate if changes were made. The images or other third party material in this article are included in the article's Creative Commons licence, unless indicated otherwise in a credit line to the material. If material is not included in the article's Creative Commons licence and your intended use is not permitted by statutory regulation or exceeds the permitted use, you will need to obtain permission directly from the copyright holder. To view a copy of this licence, visit http://creativecommons.org/licenses/by/4.0/.

\section{References}

1. Marcenes W, Kassebaum NJ, Bernabé E, Flaxman A, Naghavi M, Lopez A et al (2013) Global burden of oral conditions in 19902010: a systematic analysis. J Dent Res 92(7):592-597

2. Schiffner U, Hoffmann T, Kerschbaum T, Micheelis W (2009) Oral health in German children, adolescents, adults and senior citizens in 2005. Community Dent Health 26(1):18-22

3. Schwendicke F, Krois J, Kocher T, Hoffmann T, Micheelis W, Jordan AR (2018) More teeth in more elderly: periodontal treatment needs in Germany 1997-2030. J Clin Periodontol 45(12):14001407

4. Fries JF (1980) Aging, natural death, and the compression of morbidity. N Engl J Med 303(3):130-135

5. Saekel R (2016) New analytical tools for evaluating dental care systems - results for Germany and selected highly developed countries. Chin J Dent Res 19(2):77-88

6. Stark H, Nitschke I (2016) Krankheits- und Versorgungsprävalenzen bei Jüngeren Erwachsenen (35- bis 44-Jährige). Zahnverlust und prothetische Versorgung. In: Jordan AR, Micheelis W (eds) Fünfte
Deutsche Mundgesundheitsstudie (DMS V). IDZ-Materialienreihe. Deutscher Zahnärzte Verlag DÄV, Köln, pp 335-358

7. Petersen PE, Kandelman D, Arpin S, Ogawa H (2010) Global oral health of older people - call for public health action. Community Dent Health 27(4 Suppl 2):257-267

8. Polzer I, Schimmel M, Müller F, Biffar R (2010) Edentulism as part of the general health problems of elderly adults. Int Dent J 60(3): 143-155

9. Broadbent JM, Williams KB, Thomson WM, Williams SM (2006) Dental restorations: a risk factor for periodontal attachment loss? J Clin Periodontol 33(11):803-810

10. Moons KG, Altman DG, Reitsma JB, Collins GS (2015) Transparent reporting of a multivariate prediction model for individual prognosis or development I. new guideline for the reporting of studies developing, validating, or updating a multivariable clinical prediction model: the TRIPOD statement. Adv Anat Pathol 22(5):303-305

11. Demarco FF, Correa MB, Horta B, Barros AJ, Peres KG, Peres MA (2013) Multilevel analysis of the association between posterior restorations and gingival health in young adults: a population-based birth cohort. J Clin Periodontol 40(12):1126-1131

12. Nelson S, Albert JM, Geng C, Curtan S, Lang K, Miadich S et al (2013) Increased enamel hypoplasia and very low birthweight infants. J Dent Res 92(9):788-794

13. Micheelis W, Reich E, editors (1999) Dritte Deutsche Mundgesundheitsstudie (DMS III). Ergebnisse, Trends und Problemanalysen auf der Grundlage bevölkerungsrepräsentativer Stichproben in Deutschland 1997. Köln, Deutscher Ärzte-Verlag

14. Micheelis W, Schiffner U, editors (2006) Vierte Deutsche Mundgesundheitsstudie (DMS IV). Neue Ergebnisse zu oralen Erkrankungsprävalenzen, Risikogruppen und zum zahnärztlichen Versorgungsgrad in Deutschland 2005. Köln, Deutscher Zahnärzte Verl. DÄV 
15. Jordan AR, Micheelis W (eds) (2016) Fünfte Deutsche Mundgesundheitsstudie (DMS V). Deutscher Zahnärzte Verlag DÄV, Köln

16. Jordan AR, Bodechtel C, Hertrampf K, Hoffmann T, Kocher T, Nitschke I et al (2014) The Fifth German Oral Health Study (Fünfte Deutsche Mundgesundheitsstudie, DMS V) - rationale, design, and methods. BMC Oral Health 14(1):161

17. Clayton D, Schifflers E (1987) Models for temporal variation in cancer rates. II: Age-period-cohort models. Stat Med 6(4):469-481

18. Destatis (2012) Bevölkerung und Erwerbstätigkeit. Bevölkerungsfortschreibung auf Grundlage des Zensus 2011. Wiesbaden

19. WHO (2015) WHO global report on trends in prevalence of tobacco smoking. WHO, Geneva, p 2015

20. Hsu KJ, Yen YY, Lan SJ, Wu YM, Lee HE (2013) Impact of oral health behaviours and oral habits on the number of remaining teeth in older Taiwanese dentate adults. Oral Health Prev Dent 11(2): $121-130$

21. Gilbert GH, Duncan RP, Shelton BJ (2003) Social determinants of tooth loss. Health Serv Res 38(6 Pt 2):1843-1862

22. Ekman A (2006) Chapter 5.10: major public health problems dental health. Scand J Public Health 34(67 Suppl):139-146
23. Suominen-Taipale A-L, Alanen P, Helenius H, Nordblad A, Uutela A (1999) Edentulism among Finnish adults of working age, 19781997. Community Dent Oral Epidemiol 27(5):353-365

24. Suominen-Taipale L, Nordblad A, Vehkalahti M, Aromaa A (2008) Oral Health in the Finnish adult population. Health 2000 Survey. Helsinki

25. Österberg T, Carlsson GE, Sundh V (2009) Trends and prognoses of dental status in the Swedish population: analysis based on interviews in 1975 to 1997 by statistics Sweden. Acta Odontol Scand 58(4):177-182

26. Kassebaum NJ, Bernabé E, Dahiya M, Bhandari B, Murray CJL, Marcenes W (2014) Global burden of severe tooth loss: a systematic review and meta-analysis. J Dent Res 93(7 Suppl):20S-28S

27. Hensel E, Gesch D, Biffar R, Bernhardt O, Kocher T, Splieth CH et al (2003) Study of health in Pomerania (SHIP): a health survey in an East German region. Objectives and design of the oral health section. Quintessence Int 34(5):370-378

Publisher's note Springer Nature remains neutral with regard to jurisdictional claims in published maps and institutional affiliations. 